\title{
Molecular epidemiology of hereditary ataxia in Finland
}

\author{
Joonas Lipponen ${ }^{1,2}$, Seppo Helisalmi ${ }^{3}$, Joose Raivo ${ }^{3}$, Ari Siitonen ${ }^{1,2}$, Hiroshi Doi ${ }^{4}$, Harri Rusanen 1,2, \\ Maria Lehtilahti ${ }^{1,2}$, Mervi Ryytty ${ }^{1,2}$, Markku Laakso ${ }^{3}$, Fumiaki Tanaka ${ }^{4}$, Kari Majamaa ${ }^{1,2}$ and Laura Kytövuori ${ }^{1,2^{*}}$
}

\begin{abstract}
Background: The genetics of cerebellar ataxia is complex. Hundreds of causative genes have been identified, but only a few cause more than single cases. The spectrum of ataxia-causing genes differs considerably between populations. The aim of the study was to investigate the molecular epidemiology of ataxia in the Finnish population.

Patients and methods: All patients in hospital database were reviewed for the diagnosis of unspecified ataxia. Acquired ataxias and nongenetic ataxias such as those related to infection, trauma or stroke were excluded. Sixty patients with sporadic ataxia with unknown etiology and 36 patients with familial ataxia of unknown etiology were recruited in the study. Repeat expansions in the SCA genes (ATXN1, 2, 3, 7, 8/OS, CACNA1A, TBP), FXN, and RFC1 were determined. Point mutations in POLG, SPG7 and in mitochondrial DNA (mtDNA) were investigated. In addition, DNA from 8 patients was exome sequenced.
\end{abstract}

Results: A genetic cause of ataxia was found in 33 patients (34.4\%). Seven patients had a dominantly inherited repeat expansion in ATXN8/OS. Ten patients had mitochondrial ataxia resulting from mutations in nuclear mitochondrial genes POLG or RARS2, or from a point mutation $\mathrm{m} .8561 \mathrm{C}>\mathrm{G}$ or a single deletion in mtDNA. Interestingly, five patients were biallelic for the recently identified pathogenic repeat expansion in RFC1. All the five patients presented with the phenotype of cerebellar ataxia, neuropathy, and vestibular areflexia (CANVAS). Moreover, screening of 54 patients with Charcot-Marie-Tooth neuropathy revealed four additional patients with biallelic repeat expansion in RFC1, but none of them had cerebellar symptoms.

Conclusions: Expansion in ATXN8/OS results in the majority of dominant ataxias in Finland, while mutations in $R F C 1$ and POLG are the most common cause of recessive ataxias. Our results suggest that analysis of RFC1 should be included in the routine diagnostics of idiopathic ataxia and Charcot-Marie-Tooth polyneuropathy.

Keywords: CANVAS, Hereditary ataxia, Molecular epidemiology, Repeat expansion

\section{Background}

About $80 \%$ of the ataxias are sporadic, in which alcoholrelated ataxia and non-hereditary degenerative ataxias are the most common [1]. The majority of the hereditary ataxias are caused by dominantly inherited trinucleotide

\footnotetext{
*Correspondence: laura.kytovuori@oulu.fi

${ }^{1}$ Research Unit of Clinical Neuroscience, Medical Research Center Oulu, Oulu University Hospital and University of Oulu, P.O. Box 5000, 90014 Oulu, Finland

Full list of author information is available at the end of the article
}

repeat expansion mainly in $A T X N \mathrm{~s}$, and these ataxias form a subgroup of spinocerebellar ataxias (SCAs). In addition, ataxia is a common feature in the mitochondrial disorders caused by a defect in mitochondrial DNA or in nuclear genome.

Recessively inherited Friedreich's ataxia (FRDA) is the most frequent heritable ataxia in Southern and Western Europe, but it seems to be rather rare in Northern Europe [1-3]. Among the autosomal dominant ataxias SCA2 and SCA3 are the most prevalent 
among Caucasians $[4,5]$, whereas in the Finnish population SCA2 is very rare and SCA3 is completely absent [6]. By contrast, ATXN8/OS-related SCA8 and mitochondrial recessive ataxia syndrome (MIRAS) resulting from mutations in POLG have been reported in Finland $[6,7]$. The differences may be explained by population history, as small founder population and rapid expansion associated with geographic isolation of subpopulations have resulted in unique genetic make-up and Finnish disease heritage [8].

New sequencing techniques have enabled identification of novel ataxia-causing mutations but, unfortunately, there are almost 600 genes associated with ataxic disorders and a single gene resolves only few cases. In addition, exome sequencing does not cover intronic or intergenic regions, where disease-associated mutations may locate as well. Indeed, the most recent ataxia-causing mutation has been found in the intronic region of RFC1 gene [9, 10].

We ascertained here a population-based cohort of patiens with ataxia of unknown etiology from a defined population in northern Finland and investigated the molecular etiology of their ataxia. We characterized the genetic spectrum of ataxia and found that the pentanucleotide repeat expansion in the $R F C 1$ gene is a common cause of ataxia in Finland.

\section{Subjects and methods}

Oulu University Hospital (OUH) is the sole provider of specialized neurological services in the province of Northern Ostrobothnia with a population of 412,830 on 31 December 2019. Adult patients who had visited OUH between 1 November 1997 - 31 October 2019 and had a diagnosis of unspecified hereditary ataxia or unspecified ataxia (ICD-10: G11.9, R27.0) were identified from the OUH patient database. The search yielded 408 patients and after exclusions (see Additional file 1), a pre-visit questionnaire was mailed to 125 patients. Eighty-one patients $(64.8 \%)$ responded and five additional patients were identified during the study at the outpatient clinic. Furthermore, 11 patients were included from the cohort of 26 patients identified in a similar study covering the years 1976-1994 [11]. One patient withdrew consent leaving a total of 96 patients. Controls for RFC1 haplotyping consisted of 269 anonymous, healthy blood donors from Finnish Red Cross.

A separate cohort of polyneuropathy consisted of 66 patients with Charcot-Marie-Tooth (CMT) disease type 2, 19 patients with CMT1 and 14 patients with intermediate type CMT [12]. The screening of RFC1 was carried out in the 54 patients with CMT, who had not received a genetic diagnosis in the previous study.

\section{Molecular genetics}

DNA was extracted from the blood samples using QIAsymphony DSP DNA kit with QIAsymphony robot and QIAamp DNA Blood Kit (QIAGEN, Hilden, Germany). A flowchart describing genetic investigation is shown in Additional file 1. Briefly, a fluorescent PCR and fragment analysis was used to genotype SCA1 (ATXN1), SCA2 (ATXN2), SCA3 (ATXN3), SCA6 (CACNA1A), SCA7 (ATXN7), SCA8 (ATXN8OS), SCA17 (TBP) and $F X N$. Fragment analyses were done using a GeneScan ${ }^{\mathrm{TM}}$ LIZ500 size standard with ABI 3500xL Genetic Analyzer (Thermo Fisher Scientific, Waltham, MA, U.S.A.). The size of the fragments was determined using GeneMapper $^{\circledR} 5.0$ software (Thermo Fisher Scientific).

Common mutations in $P O L G$, the p.Ala510Val mutation in $S P G 7$, the m.3243A $>$ G and $\mathrm{m} .8344 \mathrm{~A}>\mathrm{G}$ mutations in mtDNA were examined and MT-ATP6 and MT-ATP8 genes were sequenced. The variants rs6844176, rs17584703, rs11096992 and rs2066790 defining the RFC1 haplotypes were investigated using restriction fragment length polymorphism with FastDigest $^{\circledR}$ RseI, TaaI, BseII and Eco105I (Thermo Fisher Scientific), respectively. Furthermore, the haplotypes were investigated in 269 healthy controls. Exome sequencing data were used to create a detailed haplotype associated with the pathogenic expansion (Fig. 1).

$\mathrm{XL}-\mathrm{PCR}$ of RFC1 expansion was carried as previously [9]. PhireII ${ }^{\mathrm{TM}}$ Hot Start Polymerase was used to confirm XL-PCR results with the same primers by using protocols for short and long fragments. Flanking multiplex PCR for FBN1 as a control and RFC1 was done using TaKaRa Ex Taq Hot Start ${ }^{\circledR}$ polymerase (Fig. 1). Fluorescent-labeled repeat-primed PCR-products (RP-PCR) were analyzed with a GeneScan ${ }^{\text {TM }}$ 600LIZ standard (Thermo Fisher Scientific). All PCR primers and reaction conditions are available in Additional info.

DNA library preparation for exome sequencing was carried out using Nextera Rapid Capture Exome Library kit, according to the manufacturer's protocol (Illumina, San Diego, CA, U.S.A.) in Biocenter Oulu Sequencing Center. The sequencing was done using Illumina NextSeq550 platform. Data pre-processing and quality control has been described previously [13]. The Q30 value of the contigs was an average of approximately $92.85 \%$. Variants were filtered by minor allele frequency less than $0.1 \%$. Variants in 562 ataxia-causing genes were investigated in eight patients that were exome sequenced (see Additional file 1).

\section{Results}

Ninety-six patients (48 men) from 89 families participated in the study. Median age of onset was 40 years (range, 1-75 years) and median disease duration was 


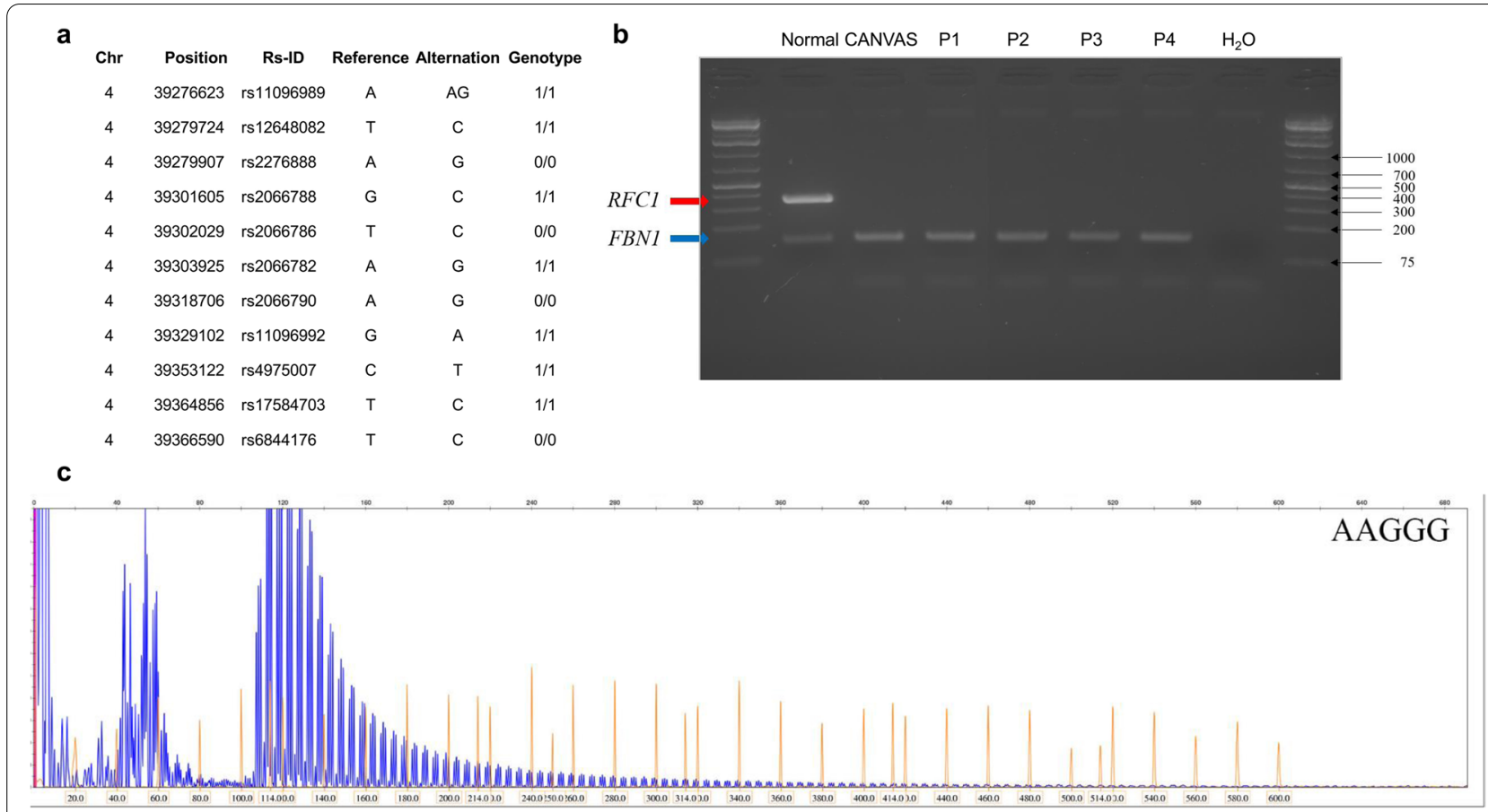

Fig. 1 RFC1 in Finnish patients with ataxia. A Core haplotype around the pathogenic expansion in the RFC1 gene. B Multiplex PCR of RFC1 normal allele and FBN1 as control in four patients with biallelic expansion. CANVAS, positive control; $\mathrm{P}$, patient; $\mathrm{H}_{2} \mathrm{O}$, negative control. C Example of repeat-primed PCR in one patient with biallelic AAGGG expansion

19 years (range, 1-68 years). A detailed family history was obtained for 89 patients, including 27 patients with ataxia in first-degree relatives.

Twenty-two patients had a previous genetic diagnosis and 11 patients received a new diagnosis (Table 1).
Clinical findings of the 33 patients with a confirmed genetic diagnosis are shown in Table 2. Expansion in ATXN8/OS (SCA8) was the most common cause of dominantly inherited ataxia, while the homozygous p.Trp748Ser mutation in POLG and the biallelic

Table 1 Genetic causes of ataxia in Finnish patients with ataxic disorders

\begin{tabular}{|c|c|c|c|}
\hline Gene & Inheritance & Mutations (Patient ID and repeat number when available) & Patients (N) \\
\hline ATXN8/OS & $A D$ & trinucleotide repeat expansion (P1 118, P2 98, P3 139, P4 190, P5 90, P6 125, P7 90) & 7 \\
\hline CACNA1A & AD & c.3414dup p.Lys1139fs (P22), c.5629-2A > G ${ }^{15}(P 23, P 24)$ & 3 \\
\hline SAMD9L & AD & c.2672 T>C p.lle891Thr & 1 \\
\hline FMRI & XLD & trinucleotide repeat expansion (P31 60) & 1 \\
\hline POLG & AR & c.2243C > G p.Trp748Ser/ c.2243C > G p.Trp748Ser & 6 \\
\hline RFC1 & AR & pentanucleotide repeat expansion $A A G G G_{\exp }$ & 5 \\
\hline SACS & AR & [c.3298G > A p.Glu1100Lys; c.4466A > G, p.Asn1489Ser]/c.4076 T>C p.Met1359Thr ${ }^{14}$ & 3 \\
\hline FXN & AR & trinucleotide repeat expansion (P25 970/970) & 2 \\
\hline RARS2 & AR & c.773G > A p.Arg258His/p.Ala369fs & 1 \\
\hline ATM & $A R$ & c.1813del p.His605fs/c.8672G > A p.Gly2891Asp & 1 \\
\hline MT-ATP6/8 & M & $\mathrm{m} .8561 \mathrm{C}>\mathrm{G}$ & 2 \\
\hline mtDNA & de novo & mtDNA deletion 4.5 kb & 1 \\
\hline No genetic diagnosis & & & 63 \\
\hline Total & & & 96 \\
\hline
\end{tabular}

GenBank reference sequences: ATM: NM_001351834.2, NP_000042.3; CACNA1A: NM_001127221.2, NP_001120693.1; mtDNA: NC_012920; POLG: NM_002693.3, NP_001119603.1; RARS2: NM_020320.5, NP_001337434.1; SACS: NM_014363.6, NP_055178.3; SAMD9L: NM_001303496.3, NP_001290425.1 
Table 2 Clinical findings in Finnish patients with genetically confirmed ataxic disorders

\begin{tabular}{|c|c|c|c|c|c|c|c|c|c|c|c|c|c|c|c|}
\hline ID & Sex & AOO & AAE & Gene & $\mathrm{Cl}$ & DM & E & $\mathrm{HI}$ & VI & PNP & Tonus & EyeM & Dysph & Dysar & KPS [18] \\
\hline 1 & $\mathrm{~F}$ & NA & NA & ATXN8/OS & - & - & - & - & - & - & NA & NA & NA & NA & NA \\
\hline 2 & $\mathrm{~F}$ & 50 & 76 & ATXN8/OS & - & - & - & + & - & - & $\mathrm{N}$ & $N$ & + & - & 80 \\
\hline 3 & F & 57 & 72 & ATXN8/OS & - & - & - & - & - & - & $\mathrm{N}$ & $\mathrm{N}$ & - & + & 80 \\
\hline 4 & $\mathrm{~F}$ & 36 & 64 & ATXN8/OS & - & - & - & - & - & - & $\mathrm{N}$ & $\mathrm{N}$ & + & + & 70 \\
\hline 5 & F & 35 & 36 & ATXN8/OS & - & - & - & - & - & - & $N$ & I & - & + & 80 \\
\hline 6 & $\mathrm{~F}$ & 33 & 36 & ATXN8/OS & - & - & - & - & - & - & $N$ & $\mathrm{~N}$ & + & + & 80 \\
\hline 7 & M & 40 & 64 & ATXN8/OS & + & + & - & - & + & + & NA & NA & + & + & 80 \\
\hline 8 & $F$ & 32 & 55 & POLG & - & - & + & - & - & + & $\mathrm{N}$ & 1 & - & + & 20 \\
\hline 9 & M & 45 & 59 & POLG & + & - & - & + & - & + & $\mathrm{N}$ & $N$ & - & - & 70 \\
\hline 10 & M & 24 & 45 & POLG & - & - & + & - & + & + & $\mathrm{N}$ & 1 & + & + & 60 \\
\hline 11 & M & 50 & 58 & POLG & - & - & - & - & - & - & N & NA & - & + & 80 \\
\hline 12 & $\mathrm{~F}$ & 30 & 43 & POLG & - & - & - & - & - & + & $\mathrm{N}$ & 1 & - & - & 80 \\
\hline 13 & $F$ & 40 & 42 & POLG & + & + & - & - & + & + & $\mathrm{N}$ & I & + & + & 80 \\
\hline 14 & $\mathrm{~F}$ & 40 & 72 & RFCl & + & - & + & - & - & + & $\mathrm{N}$ & I & + & + & 40 \\
\hline 15 & $M$ & 45 & 57 & RFCl & - & - & - & - & - & + & $\mathrm{N}$ & 1 & - & + & 60 \\
\hline 16 & $F$ & 52 & 64 & RFC1 & - & - & - & - & + & + & $\mathrm{N}$ & 1 & - & + & 70 \\
\hline 17 & $\mathrm{~F}$ & 64 & 74 & RFCl & - & + & - & - & - & + & $N$ & 1 & + & + & 50 \\
\hline 18 & M & 48 & 71 & RFCl & - & - & - & - & - & + & $\mathrm{N}$ & 1 & - & - & 90 \\
\hline 19 & $\mathrm{~F}$ & 20 & 58 & SACS & - & - & - & - & - & + & $S, R$ & 1 & - & + & 50 \\
\hline 20 & M & 10 & 65 & SACS & - & - & - & - & - & + & $S$ & I & - & + & 40 \\
\hline 21 & M & 7 & 63 & SACS & - & - & - & - & - & + & S & I & - & + & 40 \\
\hline 22 & M & 1 & NA & CACNAIA & - & - & - & - & + & - & $\mathrm{N}$ & 1 & - & + & NA \\
\hline 23 & M & 10 & 44 & CACNA1A & - & - & - & - & - & - & $\mathrm{N}$ & $N$ & - & - & 90 \\
\hline 24 & $\mathrm{~F}$ & 11 & 20 & CACNAIA & - & - & - & - & - & - & $\mathrm{N}$ & $N$ & - & - & 90 \\
\hline 25 & F & 5 & 37 & FXN & - & + & - & - & - & + & S & 1 & - & + & 50 \\
\hline 26 & $F$ & 22 & 29 & FXN & - & - & - & - & - & + & $S$ & 1 & - & - & 80 \\
\hline 27 & M & 20 & 64 & MT-ATP6/8 & - & + & - & + & - & + & $\mathrm{N}$ & $\mathrm{N}$ & + & + & 60 \\
\hline 28 & $F$ & 20 & 59 & MT-ATP6/8 & - & + & - & - & - & - & $\mathrm{N}$ & $\mathrm{N}$ & - & + & 60 \\
\hline 29 & $F$ & 32 & 38 & SAMDOL & + & - & - & - & - & - & $N$ & 1 & + & - & 80 \\
\hline 30 & M & 70 & 85 & ATM & + & - & - & - & - & - & $\mathrm{N}$ & I & + & + & 50 \\
\hline 31 & M & 57 & 63 & FMRI & + & - & - & - & - & - & $\mathrm{N}$ & $N$ & + & + & 80 \\
\hline 32 & $F$ & 2 & 28 & RARS2 & + & - & + & - & - & - & $\mathrm{N}$ & I & + & - & 70 \\
\hline 33 & M & 4 & 26 & mtDNA del & + & + & - & + & + & - & $\mathrm{N}$ & I & - & - & 40 \\
\hline
\end{tabular}

$A A E$ age at examination, $A O O$ age of onset, $C l$ cognitive impairment, del deletion, $D M$ diabetes mellitus, Dysar dysarthria, dysph dysphagia, E epilepsy, EyeM eye movements, $H$ I hearing impairment, I impaired eye movements include double vision, impaired saccades, impaired smooth pursuit or nystagmus, KPS Karnofsky performance score, $N$ normal, $N A$ not analyzed, $P N P$ polyneuropathy, R rigidity, $S$ spasticity, VI visual impairment

expansion in RFC1 were the most common causes of recessively inherited ataxia. Three patients had autosomal recessive spastic ataxia of Charlevoix-Saguenay (ARSACS) and three patients had point mutations in CACNA1A $[14,15]$. Two siblings had a mitochondrial ataxia syndrome resulting from $\mathrm{m} .8561 \mathrm{C}>\mathrm{G}$ mutation in the overlapping region of MT-ATP6 and MT-ATP8 [16]. Exome sequencing revealed a p.His605fs frameshift mutation in $A T M$ with a previously reported pathogenic mutation p.Gly2891Asp in one patient [17]. Other rare causes included a single deletion in mtDNA and mutations in SAMD9L, RARS2 and FRMI, respectively.
Axonal polyneuropathy is a core feature of CANVAS and, hence, we investigated RFC1 expansion in a separate cohort of 54 patients with a clinical diagnosis of Charcot-Marie-Tooth disease [12]. Four patients with biallelic repeat expansion were found, but none of them presented with cerebellar symptoms.

\section{RFC1 haplotypes}

The five patients with the pathogenic AAGGG expansion

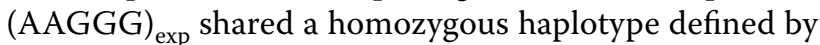
four single-nucleotide polymorphisms. The frequency of 
this haplotype was $12.8 \%$ among the patients with ataxia and $10.4 \%$ among the controls. Interestingly, two controls with the homozygous haplotype harbored a single copy of a short pathogenic expansion. A detailed haplotype created from exome sequencing data of the RFC1 patients was identical with those reported previously (Fig. 1) [10, 19].

\section{Discussion}

We found that the $A T X N 8 / O S$ trinucleotide repeat expansion is the most prevalent cause of dominantly inherited ataxia in Finland confirming a previous observation [6]. Common recessive causes were p.Trp748Ser in POLG and the recently discovered intronic expansion in RFC1. SCA3, FRDA and SPG7-related ataxia have been reported to be the most common hereditary ataxias among Europeans [4, 20], but we found only two patients with FRDA and none with SCA3 or mutations in SPG7. Altogether, $34 \%$ of our patients received a genetic diagnosis and the three most common genetic causes accounted for half of the diagnoses. These frequencies are specific to Finns and emphasize genetic differences between Caucasian populations.

CANVAS is a new member in the syndromes that are caused by an intronic mutation $[9,10]$. The biallelic expansion (AAGGG) exp $_{\text {in }} R F C 1$ was found in five patients that presented with ataxia, polyneuropathy and nystagmus resembling typical CANVAS [21]. Vestibular dysfunction is a core feature in CANVAS and, consistently, our patients had abnormal eye movements, but vestibular areflexia had been confirmed with caloric test only in one patient. Another patient had a more severe phenotype including epilepsy and cognitive impairment. Neither epilepsy nor cognitive impairment are features in typical CANVAS, but atypical phenotypes, such as dementia with Lewy bodies, have been reported recently [19]. The repeat expansion in RFC1 is a new genetic cause of disease and it is probable that new features of the phenotype will be discovered. In addition, we found four patients with biallelic repeat expansion in RFC1 among patients with Charcot-Marie-Tooth disease [12]. These numbers suggest that RFC1 is among the three most common genetic causes of Charcot-Marie-Tooth disease in Finland together with PMP22 duplication and p.His123Arg in GDAP1.

Two controls with the homozygous RFC1 haplotype harbored a single copy of a short pathogenic expansion, but the risk of developing a large expansion is unknown for those who harbor a short form of the expansion. All subjects are potential carriers of the large expansion, if they harbor one or two copies of the haplotype that is associated with the large repeat expansion. However, the large expansion allele cannot be amplified in PCR and thus, heterozygous subjects may appear as homozygous for the normal-sized repeat allele even if they, in fact, are carriers of the large expansion. Segregation analyses with determination of the exact size of the expansion are required in order to estimate the risk of having an increase in the repeat number in the next generation.

A mitochondrial cause of ataxia was detected in ten patients including six patients with homozygous mutations in $P O L G$, siblings with $\mathrm{m} .8561 \mathrm{C}>\mathrm{G}$ [16], a patient with a single deletion in mtDNA and a patient with compound heterozygous mutations in RARS2. Recently, the $\mathrm{m} .8561 \mathrm{C}>\mathrm{T}$ mutation has been reported in a patient with severe mitochondrial disorder [22] suggesting that MT-ATP genes may be mutational hotspots in the aetiology of ataxia. Mitochondrial defects have previously been found in $>20 \%$ of ataxia patients, especially in those with a syndromic phenotype [23]. Single patients were found to harbour mutations in ATM, SAMD9L, or FMRI that have occasionally been reported as a cause of inherited ataxia [24-26]. The mutation in ATM was found in exome sequencing, but the genetic cause remained unclear in the seven other patients that were analyzed for 562 ataxia-causing genes.

\section{Conclusions}

We found that mutations in ATXN8/OS, POLG and RFC1 are the most common genetic causes of ataxia in Finland. The pentanucleotide repeat expansion in RFC1 is a frequent cause of polyneuropathy as well suggesting that routine diagnostic testing should be carried out in patients with suspected hereditary ataxia or polyneuropathy.

\section{Abbreviations \\ CANVAS: Cerebellar ataxia, neuropathy, and vestibular areflexia; FRDA: Friedreich's ataxia; MIRAS: Mitochondrial recessive ataxia syndrome; mtDNA: Mitochondrial DNA; OUH: Oulu University Hospital; RP-PCR: Repeat-primed polymerase chain reaction; SCA: Spinocerebellar ataxia.}

\section{Supplementary Information}

The online version contains supplementary material available at https://doi. org/10.1186/s12883-021-02409-z.

Additional file 1: Figure 1. Selection criteria of the patients. Figure 2. Genetic investigations of the patients. Table 1. List of 562 genes analyzed from exome sequencing data.

Additional file 2: Additional info. Lab protocols.

Acknowledgements

We thank Mrs. Anja Heikkinen for her technical expertise.

\section{Authors' contributions}

$J \mathrm{~L}$ collected the samples and analyzed the clinical data. SH, JR and ML provided the analyses of the ATXN1-3, 7, 8/OS, CACNA1A, TBP and FXN genes. $M R, M L$ and $H R$ participated in the clinical analyses. AS did the analyses of raw exome sequencing data. HD and FT did the flanking PCR and RP-PCR of RFC1. 
KM planned the study, collected the samples and participated in the clinical analyses. LK did the molecular genetics of RFC1 and point mutations, analyzed the exome sequencing data and wrote the first draft of the manuscript. All authors revised and approved the final manuscript.

\section{Funding}

The study was funded by grants from the Sigrid Jusélius Foundation and from the Medical Research Center Oulu. The study received state research funding from Oulu University Hospital. The funders did not have any influence on the results of this study.

\section{Availability of data and materials}

The phenotype-genotype data of the study are available in the manuscript. The lab protocols are available in the additional info of the manuscript. The mtDNA sequences are available in the GenBank with accession numbers MZ475290-MZ475297. Variants have been submitted to ClinVar (identifiers pending) and MitoMap (https://www.mitomap.org/MITOMAP). Due to privacy policies, the exome sequencing data cannot be publicly available.

\section{Declarations}

\section{Ethics approval and consent to participate}

The study protocol was approved by the Oulu University Hospital Ethics Committee (EETTMK:93/2010). All participants gave their informed consent for genetic analyses and for reporting the results. All clinical examinations were done according the WMA declaration of Helsinki.

\section{Consent for publication}

Not applicable.

\section{Competing interests}

The authors declare that they have no competing interests.

\section{Author details}

${ }^{1}$ Research Unit of Clinical Neuroscience, Medical Research Center Oulu, Oulu University Hospital and University of Oulu, P.O. Box 5000, 90014 Oulu, Finland. ${ }^{2}$ Department of Neurology, Oulu University Hospital, Oulu, Finland. ${ }^{3}$ Institute of Clinical Medicine, Internal Medicine, University of Eastern Finland, Kuopio, Finland. ${ }^{4}$ Department of Neurology and Stroke Medicine, Yokohama City University Graduate School of Medicine, Yokohama, Japan.

Received: 17 March 2021 Accepted: 17 September 2021 Published online: 02 October 2021

\section{References}

1. Hadjivassiliou M, Martindale J, Shanmugarajah P, Grünewald RA, Sarrigianis PG, Beaucamp N, et al. Causes of progressive cerebellar ataxia: prospective evaluation of 1500 patients. J Neurol Neurosurg Psychiatry. 2017:88(4):301-9.

2. Juvonen V, Kulmala SM, Ignatius J, Penttinen M, Savontaus ML. Dissecting the epidemiology of a trinucleotide repeat disease-example of FRDA in Finland. Hum Genet. 2002;110(1):36-40.

3. Vankan P. Prevalence gradients of Friedreich's Ataxia and R1b haplotype in Europe co-localize, suggesting a common Palaeolithic origin in the Franco-Cantabrian ice age refuge. J Neurochem. 2013;126(Suppl 1):11-20.

4. Ruano L, Melo C, Silva MC, Coutinho P. The global epidemiology of hereditary ataxia and spastic paraplegia: a systematic review of prevalence studies. Neuroepidemiology. 2014;42(3):174-83.

5. Salman MS. Epidemiology of cerebellar diseases and therapeutic approaches. Cerebellum. 2018;17(1):4-11.

6. Juvonen V, Hietala M, Kairisto V, Savontaus ML. The occurrence of dominant spinocerebellar ataxias among 251 Finnish ataxia patients and the role of predisposing large normal alleles in a genetically isolated population. Acta Neurol Scand. 2005;111(3):154-62.

7. Hakonen AH, Heiskanen S, Juvonen V, Lappalainen I, Luoma PT, Rantamäki, et al. Mitochondrial DNA polymerase W748S mutation: a common cause of autosomal recessive ataxia with ancient European origin. Am J Hum Genet. 2005;77(3):430-41.
8. Peltonen L, Jalanko A, Varilo T. Molecular genetics the Finnish disease heritage. Hum Mol Genet. 1999;8(10):1913-23.

9. Cortese A, Simone R, Sullivan R, Vandrovcova J, Tariq H, Yau WY, et al, Biallelic expansion of an intronic repeat in RFC1 is a common cause of late-onset ataxia. Nat Genet. 2019;51(4):649-58.

10. Rafehi H, Szmulewicz DJ, Bennett MF, Sobreira NL, Pope K, Smith KR, et al. Bioinformatics-Based Identification of Expanded Repeats: A Non-reference Intronic Pentamer Expansion in RFC1 Causes CANVAS. Am J Hum Genet. 2019;105(1):151-65.

11. Majamaa K, Moilanen JS, Uimonen S, Remes AM, Salmela PI, Kärppä M, et al. Epidemiology of A3243G, the mutation for mitochondrial encephalomyopathy, lactic acidosis, and strokelike episodes: prevalence of the mutation in an adult population. Am J Hum Genet. 1998;63(2):447-54.

12. Marttila M, Kytövuori L, Helisalmi S, Kallio M, Laitinen M, Hiltunen M, et al. Molecular epidemiology of Charcot-Marie-Tooth disease in Northern Ostrobothnia, Finland: a population-based study. Neuroepidemiology. 2017:49(1-2):34-9.

13. Siitonen A, Nalls MA, Hernández D, Gibbs JR, Ding J, Ylikotila P, et al. Genetics of early-onset Parkinson's disease in Finland: exome sequencing and genome-wide association study. Neurobiol Aging. 2017;53:195e7-10.

14. Palmio J, Kärppä M, Baumann P, Penttilä S, Moilanen J, Udd B. Novel compound heterozygous mutation in SACS gene leads to a milder autosomal recessive spastic ataxia of Charlevoix-Saguenay, ARSACS, in a Finnish family. Clin Case Rep. 2016;4(12):1151-6.

15. Kaunisto MA, Harno $H$, Kallela $M$, Somer $H$, Sallinen $R$, Hämäläinen $E$ et al. Novel splice site CACNA1A mutation causing episodic ataxia type 2. Neurogenetics. 2004;5(1):69-73.

16. Kytövuori L, Lipponen J, Rusanen $\mathrm{H}$, Komulainen T, Martikainen MH, Majamaa K. A novel mutation m. 8561C> G in MT-ATP6/8 causing a mitochondrial syndrome with ataxia, peripheral neuropathy, diabetes mellitus, and hypergonadotropic hypogonadism. J Neurol. 2016;263(11):2188-95.

17. Byrd PJ, Srinivasan V, Last JI, Smith A, Biggs P, Carney EF, et al. Severe reaction to radiotherapy for breast cancer as the presenting feature of ataxia telangiectasia. Br J Cancer. 2012;106(2):262-8.

18. Karnofsky DA, Abelmann WH, Craver LF, Burchenal JH. The use of the nitrogen mustards in the palliative treatment of carcinoma. With particular reference to bronchogenic carcinoma. Cancer. 1948;1 (4):634-56.

19. Nakamura H, Doi H, Mitsuhashi S, Miyatake S, Katoh K, Frith MC, et al. Longread sequencing identifies the pathogenic nucleotide repeat expansion in RFC1 in a Japanese case of CANVAS. J Hum Genet. 2020;65(5):475-80.

20. Choquet K, Tétreault M, Yang S, La Piana R, Dicaire MJ, Vanstone MR, et al. SPG7 mutations explain a significant proportion of French Canadian spastic ataxia cases. Eur J Hum Genet. 2016;24(7):1016-21.

21. Cortese A, Tozza S, Yau WY, Rossi S, Beecroft SJ, Jaunmuktane Z, et al. Cerebellar ataxia, neuropathy, vestibular areflexia syndrome due to RFC1 repeat expansion. Brain. 2020;143(2):480-90.

22. Fragaki K, Chaussenot A, Serre V, Acquaviva C, Bannwarth S, Rouzier C, et al. A novel variant m. 8561C> T in the overlapping region of MT-ATP6 and MT-ATP8 in a child with early-onset severe neurological signs. Mol Genet Metab Rep. 2019;21:100543.

23. Bargiela D, Shanmugarajah P, Lo C, Blakely EL, Taylor RW, Horvath R, et al. Mitochondrial pathology in progressive cerebellar ataxia. Cerebellum Ataxias. 2015;2:16.

24. Savitsky K, Bar-Shira A, Gilad S, Rotman G, Ziv Y, Vanagaite L, et al. A single ataxia telangiectasia gene with a product similar to PI-3 kinase. Science. 1995;268(5218):1749-53.

25. Chen DH, Below JE, Shimamura A, Keel SB, Matsushita M, Wolff J, et al. Ataxia-pancytopenia syndrome is caused by missense mutations in SAMD9L. Am J Hum Genet. 2016;98(6):1146-58.

26. Salcedo-Arellano MJ, Cabal-Herrera AM, Tassanakijpanich N, McLennan YA, Hagerman RJ. Ataxia as the Major Manifestation of Fragile X-Associated Tremor/Ataxia Syndrome (FXTAS): Case Series. Biomedicines. 2020;8(5):136.

\section{Publisher's Note}

Springer Nature remains neutral with regard to jurisdictional claims in published maps and institutional affiliations. 